\title{
Fall Prevention in Community-Dwelling Adults with Mild to Moderate Cognitive Impairment: A Systematic Review and Meta-Analysis
}

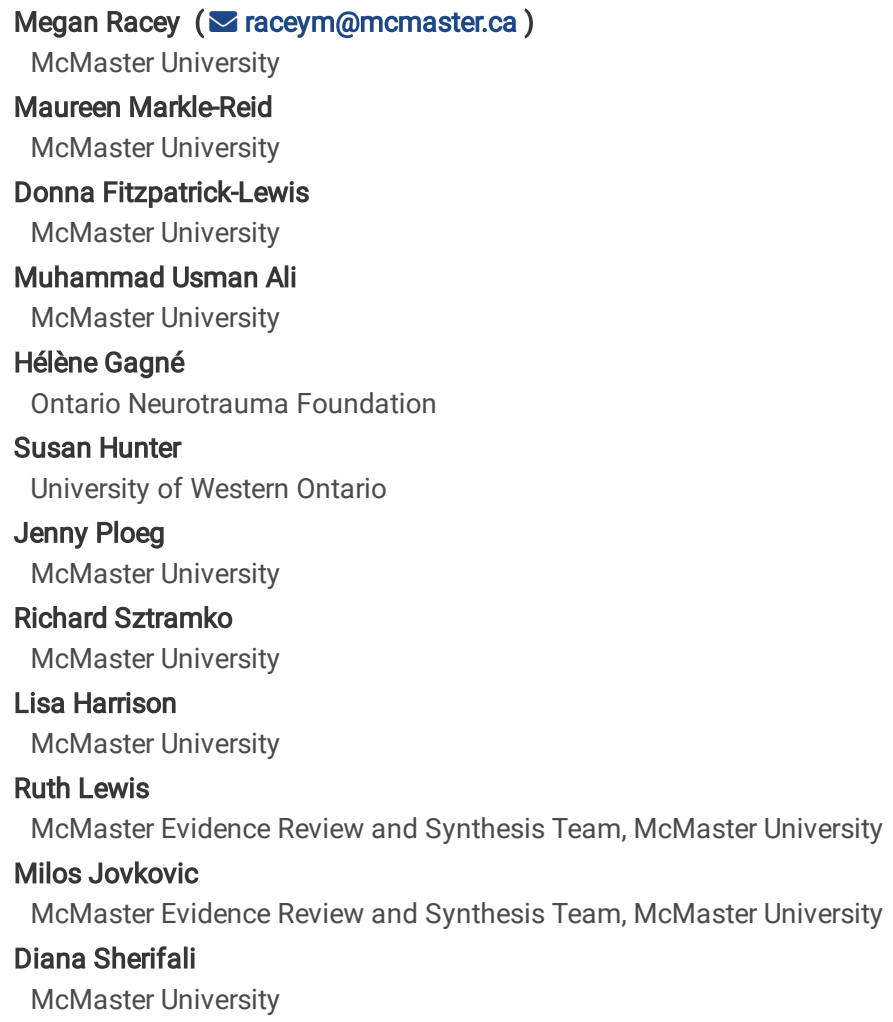

Version of Record: A version of this preprint was published at BMC Geriatrics on December 1st, 2021. See the published version at https://doi.org/10.1186/s12877-021-02641-9. 


\section{Abstract}

Background: Cognitive impairment $(\mathrm{Cl})$ increases an individual's risk of falls due to the role cognition plays in gait control. Older adults with dementia fall 2-3 times more than cognitively healthy older adults and $60-80 \%$ of people with dementia fall annually. Practitioners require evidence-based fall prevention best practices to reduce the risk of falls in cognitively impaired adults living in the community.

Methods: We conducted a systematic review and meta-analysis to identify the effectiveness of primary and secondary fall prevention interventions in reducing falls and fear of falling, and improving gait, balance, and functional mobility. We searched 7 databases for fall prevention interventions involving communitydwelling adults $\geq 50$ years with mild to moderate $\mathrm{Cl}$. Reviewers screened citations, extracted data, and assessed risk of bias and certainty of evidence (GRADE). We assessed statistical and methodological heterogeneity and performed a meta-analysis of studies including subgroup analysis based on intervention and risk of bias groupings.

Results: 509 community-dwelling adults (mean age 67.5 to 84.0 years) with mild to moderate $\mathrm{Cl}$ from 12 randomized or clinical controlled trials (RCTs/CCTs) were included in this review. Eight studies were exercise interventions, 3 were multifactorial, and 1 provided medication treatment. Fall prevention interventions had significant effects of medium magnitude on fear of falling (standardized mean difference (SMD) -0.73 [-1.10, -0.36$]$ ), balance (SMD 0.66 [0.19, 1.12]), and functional mobility measured as Timed Up and Go test (SMD -0.56 [-0.94, -0.17]) and significant effects of small magnitude on gait control (SMD 0.26 [0.08, 0.43]) all with moderate certainty of evidence. The meta-analysis showed no significant effects for falls (number of events or falls incidence). Sub-analysis showed that exercise and low risk of bias studies remained significant for balance and perceived risk of falls.

Conclusion: The effect of fall prevention interventions on direct outcomes, such as falls, remains unclear in cognitively impaired individuals. Exercise interventions are effective at improving fall risk factors, however, high quality studies with longer follow-up and adequate sample sizes are needed to determine their effectiveness on falls directly. There remains a gap in terms of effective fall prevention interventions for older adults with $\mathrm{Cl}$.

\section{Background}

Falls affect more than $30 \%$ of the adult population aged 65 years and older (1) and can result in negative health outcomes and severe injuries such as traumatic brain injuries and even death (2). Falls in older adults are multifactorial with over 400 identifiable risk factors such as physical deficits, reduced lower-limb strength, gait and balance impairment, previous slips or trips, difficulty in activities of daily living (ADLs), functional impairment, prescribed drugs and medications, environmental hazards, and others (3-5). Not only can falls result in injuries, but they can also lead to negative mental health outcomes such as fear of falling, loss of autonomy, poor quality of life, greater isolation, confusion, and depression. Falls also cost our public health system as they are the leading cause of injury-related admissions to acute care hospitals and in-hospital deaths. With an aging population the cost of fall injuries to seniors in Canada is estimated to rise from $\$ 2.4$ billion a year in direct healthcare costs (2) to $\$ 240$ billion by 2040 (4).

Cognitive impairment $(\mathrm{Cl})$ is an important risk factor for experiencing falls. Any adult with cognitive impairment can also experience falls at an increased rate compared to the general population that is cognitively healthy (5). This is due to the role cognition plays in the control of gait and the altered gait patterns that adults with cognitive impairment demonstrate (3). In fact, older adults with dementia fall two to three times more than cognitively healthy older adults (6) and $60-80 \%$ of people with dementia fall annually (3). Cognitive impairment occurs on a continuum from mild, moderate, to severe states. Mild cognitive impairment $(\mathrm{MCl})$ is an intermediate clinical state between normal cognitive aging and dementia, and it precedes and leads to dementia in many cases (7). It is defined by subjective cognitive complaints and measurable cognitive decline in the absence of interference with daily function (8). The term dementia is used when individuals have subjective cognitive complaints, measurable cognitive decline, and functional impairment. The terms Mild or Moderate Dementia are used when employing functional or cognitive assessments to distinguish where along the spectrum from mild to severe cognitive impairment (or dementia) an individual may exist. Cognitive impairment is often due to neurodegenerative diseases that are associated with advancing age, but it can also occur due to head trauma, stroke, or other diseases and affect those at any age.

Fall prevention strategies have demonstrated success in reducing risk factors and rate of falls in the cognitively healthy older adults (9); however, these practices have not translated successfully to adults with cognitive impairment. There are limited reviews which include meta-analyses on interventions to specifically help adults with cognitive impairment in a community-based setting, despite these people having a disproportionately high rate of falls and fractures and poorer outcomes after falls $(10,11)$. Published reviews are either focused on a general older adult population (12) or on specific intervention types such as physical activity $(13,14)$. Previous reviews which have focused on cognitively impaired individuals have concentrated on more mixed populations (including many types of $\mathrm{Cl}$ ) and settings, primarily on institutionalized facilities such as hospitals and long-term care homes $(3,15)$.

Based on practical work with relevant stakeholders, including members of the LOOP Fall Prevention Community of Practice (16) it has also become clear that there is a need to better translate research into practice. Stakeholders, including practitioners, are requesting evidence for interventions and best practices to implement in order to reduce falls in adults with cognitive impairment who are living in the community. While there are existing fall prevention guidelines (17) they are not specific to community-dwelling and/or cognitively impaired adults. Stakeholders are struggling to find this information and are in need of actionable, evidence-based guidelines and recommendations for practice.

Our review will directly address these gaps in evidence and practice by identifying the effectiveness of primary and secondary fall prevention interventions in community-dwelling adults with mild to moderate cognitive impairment. This will support future work which builds upon gaps in the research and addressing the barriers and facilitators of implementing fall prevention activities in the community $(8,9)$. The outcomes of this review may have implications for research, practice, and policy.

\section{Methods}


This systematic review and meta-analysis followed the Preferred Reporting Items for Systematic Reviews and Meta-analyses (PRISMA) guidelines (18) and reports on the outcomes ranked critical by our interdisciplinary research team (H.G., S.W.H., M.M.R., J.P., L.H., R.S., D.S.) from a registered protocol (PROSPEROCRD42020210916). Our methods follow the Cochrane Handbook for Systematic Reviews of Interventions Version 6, 2019 (19).

\section{Search Strategy}

The search terms, databases, and strategy were developed in consultation with a research librarian at McMaster University and informed by previous systematic reviews $(11,12,15)$ (Additional File 1). We searched MEDLINE, Embase, PsycINFO, Cochrane Central Register of Controlled Trials (CENTRAL), Cumulative Index of Nursing and Allied Health Literature (CINAHL), Web of Science, and Science Direct up to April 2020 and manually searched reference lists of relevant reviews and included studies for citations not captured in our search. Results from the search were deduplicated, and citations were uploaded to a secure internet-based platform for screening (DistillerSR, Evidence Partners Inc., Ottawa, Canada).

\section{Eligibility Criteria}

We included fall prevention interventions in community-dwelling adults (aged 50+) with mild and/or moderate $\mathrm{Cl}$. $\mathrm{Cl}$ had to be assessed by a valid and reliable tool, diagnosis or medical report, and/or clearly identified and described by study authors. Studies with general adult populations or mixed populations but which have subgroup analysis for participants with mild or moderate cognitive impairment, were also considered. Without subgroup analysis, a mixed population must have at least $80 \%$ of participants with our targeted condition (mild to moderate cognitive impairment) to be included in our review. For our review, community dwelling included individuals living in a community setting (with or without caregiver support) and can include different locations/settings, however, we excluded those living in retirement homes, nursing homes, long-term care homes, acute care, or hospital settings where they may receive full-time support and care for activities of daily living. We also excluded studies of older adults with severe $\mathrm{Cl}$.

The main purpose of the intervention had to be either primary or secondary fall prevention as defined by the Institute for Work and Health (20). Briefly, primary fall prevention aims to prevent a fall before it ever occurs whereas secondary fall prevention aims to reduce the number and severity of falls in those who have already experienced falls. Studies must have been available in English, peer-reviewed, and comprised of interventions with a control group (randomization was not required). For our review, a control group was defined as treatment as usual, usual care (i.e., no change to usual activities), or minimal contact (an intervention not thought to reduce falls such as general health education or social visits).

Outcomes were not used for inclusion or exclusion of studies. Outcomes of interest were selected by our interdisciplinary research team (H.G., S.W.H., M.M.R., J.P., L.H., R.S., D.S.) through an anonymized voting process. This process involved gathering a comprehensive list of outcomes and their associated tools/measurements based on our research and clinical expertise, factors related to falls risk (4), and existing and relevant systematic reviews (11, 12, 15). The team identified any missing outcomes and then anonymously ranked the outcomes on a scale of 1-9 (<4 not important, 4-6 important, 7-9 critical) based on Grading of Recommendation, Assessment, Development and Evaluations (GRADE) methodology (21). Those not involved in the ranking process (D.F.L., M.R.) compiled and averaged the scores for each outcome and provided the list of outcomes to the committee for final discussion and agreement. Outcomes with an average score that reached critical were included in this review and consisted of Falls, Perceived Risk of Falls/Fear or Concern of Falls, Balance, Other fall measures (such as slips and trips), Gait Speed and Control, Functional Mobility, and Mortality. The full list of outcomes, their definitions, and the tools used to measure these outcomes can be found in Additional File 2.

\section{Data Extraction and Quality Assessment}

A team of researchers conducted the screening and data extraction (M.R., D.F.L., R.L., M.J.). A minimum of two reviewers were required to independently and in duplicate screen titles and abstracts of all potentially eligible studies. Articles marked for inclusion by any team member went on to full-text screening which was completed independently and in duplicate by 2 team members and required consensus for inclusion or exclusion. We developed, piloted, and deployed standardized forms for data extraction. Two team members independently completed full data extraction of study characteristics (setting, sample size, inclusion and exclusion criteria, characteristics of participants, type of intervention (categories based on existing literature (22) and taxonomies (23)), and experimental and control components) and intention-to-treat data for the outcomes listed above. In cases where studies had multiple measures for the same outcome, we extracted the primary or direct measures before using secondary outcomes or subgroup analysis data (i.e., within the same study, gait speed was preferred over dual-task cost measures). Reviewers also assessed study risk of bias (RoB) using the Cochrane Collaboration RoB tool (24) for randomized controlled trials (RCTs). All extraction was independently verified by the statistician (M.A.). Conflicts were resolved by the lead researcher of this review (M.R.).

We independently evaluated the certainty of the body of evidence using the Grading of Recommendation, Assessment, Development and Evaluations (GRADE) method (25) with GRADEpro software (26). GRADE rates the certainty of a body of evidence as high, moderate, low, or very low and ratings are based on an assessment of 5 conditions: 1 . methodological quality, 2. consistency across effect estimates/statistical heterogeneity, 3 . directness of the body of evidence to the populations, interventions, comparators and/or outcomes of interest, 4. precision of results, and 5. indications of reporting bias.

\section{Statistical Analysis}

All data analyses were planned a priori. A meta-analysis was used to combine the results across studies by outcome using the published data from included studies. For the binary outcomes, we utilized the number of events; proportion or percentage data to generate the summary measures of effect in the form of risk ratio (RR) using DerSimonian and Laird random effects models with inverse variance method (27).

For continuous outcomes, we used immediate post-treatment data (means, standard deviations of change from baseline scores). The DerSimonian and Laird random effects models with inverse variance (IV) method were used to generate the summary measures of effect in the form of mean difference (MD) (27). For instances where multiple outcome measures were used for the same outcome, we generated the summary measures of effect in the form of standardized mean differences (SMD) (27). The SMD was used as a summary statistic because the studies in this systematic review often assessed the same outcome 
measured in a variety of ways (e.g., gait measured as gait speed, stride length, stride time, 6 metre walk test (6MWT), use of gait aids, step tests, Dynamic Gait Index (DGI), coefficient of variation, etc.). In this situation, it was necessary to standardize the results of the studies before they could be compared across studies or combined in a quantitative synthesis. The SMD based effect sizes represent the magnitude of intervention effect relative to the variability observed within a particular study. Therefore, the studies for which the difference in mean change score was the same as the proportion of standard deviation of mean change score will have the same SMD, regardless of the actual scale or unit of measurement used to assess the outcome measures $(28,29)$. The SMD is interpreted based on its magnitude according to Cohen $d$ recommended thresholds $(\sim 0.2=$ small effect, $\sim 0.5=$ medium effect, $\sim 0.8=$ large effect) $(30)$. For studies where measure of variance was reported as confidence intervals, standard error, or $\mathrm{p}$-values, we used Cochrane recommended methods to convert this data to standard deviation (28).

We used a random effects multi-level meta-analytic approach to account for dependency between effect sizes (i.e., the correlation between effect sizes due to multiple measures or sub-measures of the same outcome within a study or comparison of multiple interventions to a single control group). In such cases, multiple measures and comparisons from the same study were nested within studies first and variance in observed effect sizes was decomposed into sampling variance, within study variance and between-study variance to account for intracluster (or intraclass) correlation in the true effects (29, 31$)$. For pooling of performance measures, the direction of effect was adjusted to ensure consistency of desirable outcome responses (i.e., reduction in gait speed measured in seconds to cover a standard distance reflects a better outcome, whereas an increase in gait speed measured in meters per second reflects a positive outcome).

We conducted further subgroup and meta-regression analysis based on fall prevention intervention type and study quality (risk of bias) where possible (for outcomes where there were more than 5 studies).

The Cochran's $Q(a=0.05)$ was employed to detect statistical heterogeneity and $I^{2}$ statistic to quantify the magnitude of statistical heterogeneity between studies where $\mathrm{I}^{2}>50 \%$ represents moderate and $\mathrm{I}^{2}>75 \%$ represents substantial heterogeneity across studies $(29,32)$. The statistical heterogeneity $\mathrm{I}^{2}$ statistic was estimated in the context of multi-level meta-analytical approach i.e. within-cluster heterogeneity (i.e. across effect sizes or multiple arms from same study) and between-cluster heterogeneity (i.e. effect sizes across studies or subgroups of interest) $(29,31)$. Overall $I^{2}$ for each summary effect size was estimated to represent the heterogeneity not attributable to sample error and is the sum of within-cluster and between-cluster heterogeneity. All analyses were performed using R software (metafor (33) and dmetar (34) packages).

\section{Results}

From 20,727 citations, we assessed 479 full-text articles for eligibility, and included 10 RCTs and 2 clinical controlled trials (CCTs) in this review (Fig. 1) (35). The majority of studies $(n=8)$ were exercise interventions $(35-42)$, while 3 were multifactorial $(10,43,44)$, and 1 provided medication treatment $(45)$. All studies included one intervention/treatment group and one control group, except for one study which tested two versions of the same intervention at different intensities (high or moderate intensity) compared to one control group (36). The studies were published from 2010 to 2020 . Further details of the included studies can be found in Table 1 and demographic data from studies can be found in Additional File 3. A total sample of 509 community-dwelling adults with mild to moderate cognitive impairment were included in this review with a mean age ranging from 67.5 to 84.0 years and percentage of women in the studies ranging from $20-74 \%$. All included studies had fewer than 122 participants with most studies consisting of less than 50 participants total $(n=8)$. Attrition rates ranged greatly from $4.5 \%$ (10) to $71.4 \%$ (35); however, most studies had attrition rates in the ranges of 9-20\% (Additional File 3). Studies were conducted across the globe in North America, South America, Europe, Asia, and Australia, and intervention duration was between 4 weeks to one year, but most studies ( $\mathrm{n}$ = 8) were between 12 weeks and 6 months in duration.

The majority of studies $(n=10)$ used the Mini Mental State Exam (MMSE), or some variation, for assessing their participants' cognitive impairment. Four studies used the Montreal Cognitive Assessment (MoCA) tool. These tools were also used for inclusion of participants based on specified cut-off scores, which varied depending on the study. Formal diagnosis of mild to moderate cognitive impairment, dementia, or Alzheimer's Disease by a physician or qualified standard such as Petersen's criteria (46) or the DSM-IV: Diagnostic and Statistical Manual of Mental Disorders (47), was also considered for inclusion of participants in 11 of the studies. Seven of the included studies specifically stated there were no serious adverse events related to the intervention, 3 made no mention of adverse events, and 3 reported some minor adverse events due to the intervention such as stiffness, mild joint pain, or were unclear if the adverse events were intervention-related. 
Table 1

Demographics and Characteristics of Fall Prevention Intervention Included Studies

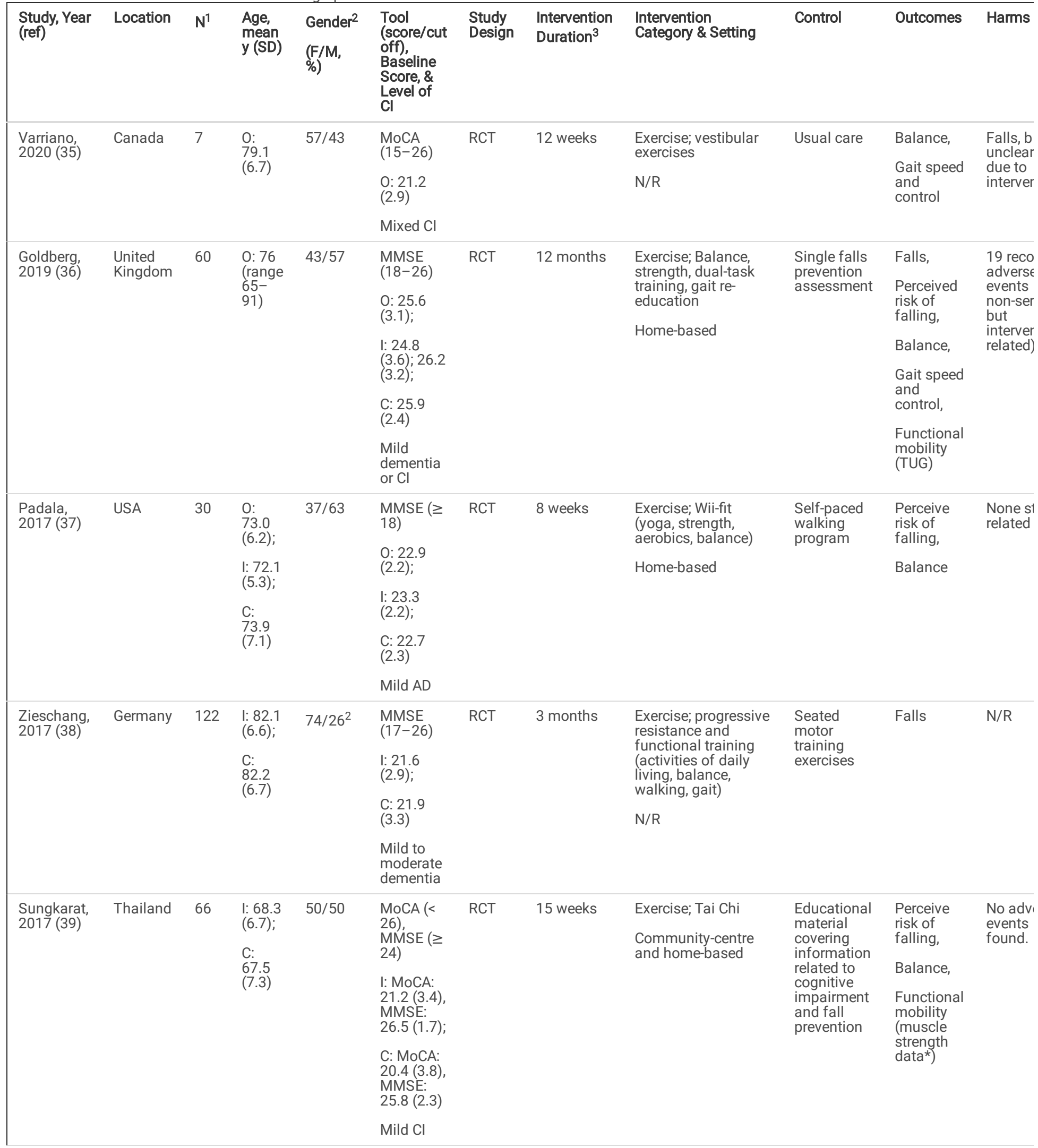

$\mathrm{Cl}=$ cognitive impairment; $\mathrm{O}=$ overall population; $\mathrm{I}=$ intervention; $\mathrm{C}=$ control; $\mathrm{AD}=$ Alzheimer's Disease; $\mathrm{N} / \mathrm{R}=$ not reported; $\mathrm{RCT}=$ randomized controlled trial; $\mathrm{C}$ = clinical (non-randomized) controlled trial. MoCA = Montreal cognitive assessment (Score /30); MMSE = Mini Mental State Exam; ACE-R = Addenbrooke's cognitive examination - revised; $C D R=$ Clinical Dementia Rating scale; $T U G$ = timed up and go test; $C S T$ = chair sit stand test; $F R T=$ functional reach test 


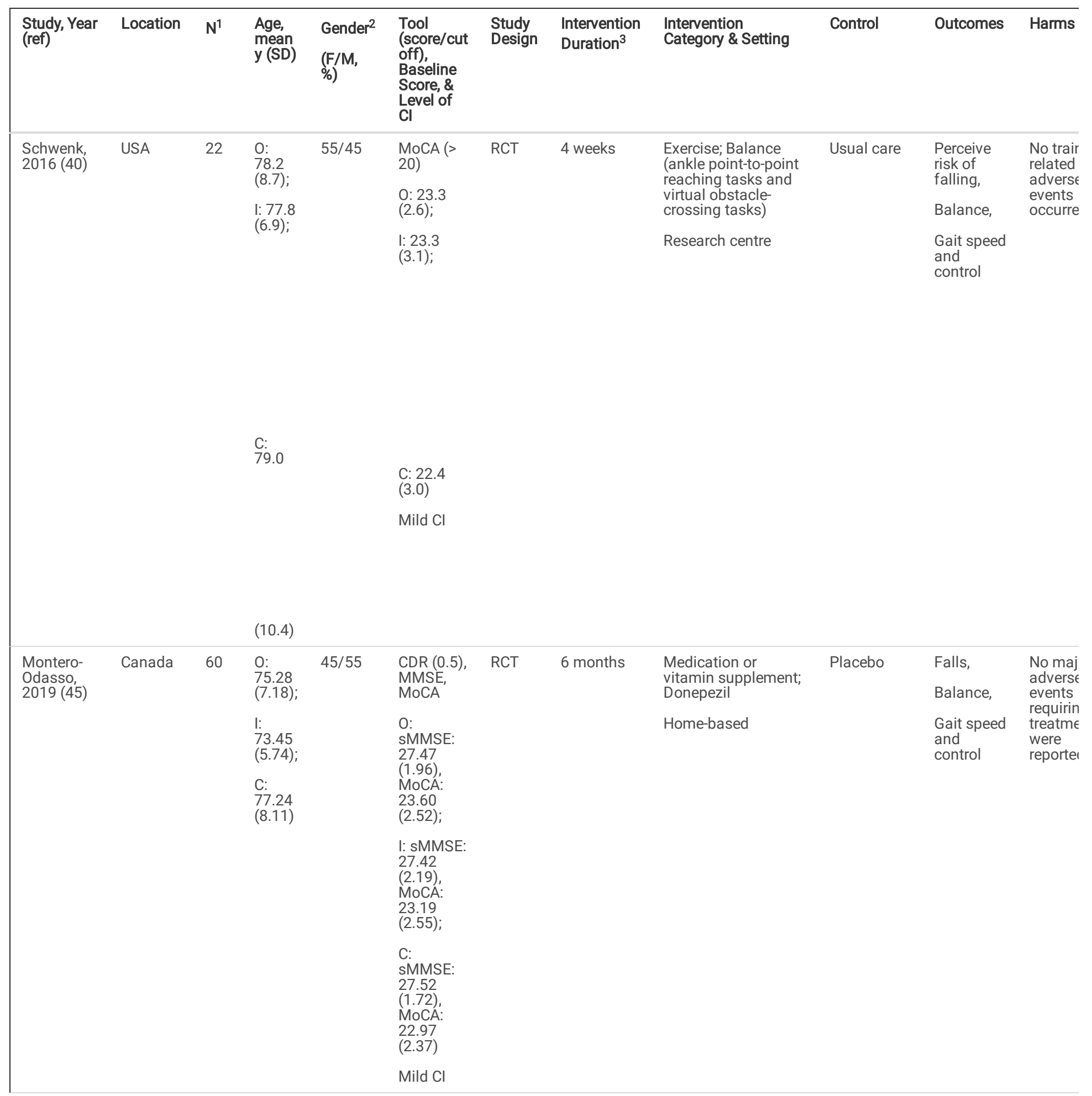

$\mathrm{Cl}=$ cognitive impairment; $\mathrm{O}=$ overall population; $\mathrm{I}=$ intervention; $\mathrm{C}=$ control; $\mathrm{AD}=$ Alzheimer's Disease; $\mathrm{N} / \mathrm{R}=$ not reported; $\mathrm{RCT}=$ randomized controlled trial; $\mathrm{C}$ $=$ clinical (non-randomized) controlled trial. MoCA = Montreal cognitive assessment (Score /30); MMSE = Mini Mental State Exam; ACE-R = Addenbrooke's cognitive examination - revised; CDR = Clinical Dementia Rating scale; TUG = timed up and go test; CST = chair sit stand test; FRT = functional reach test 


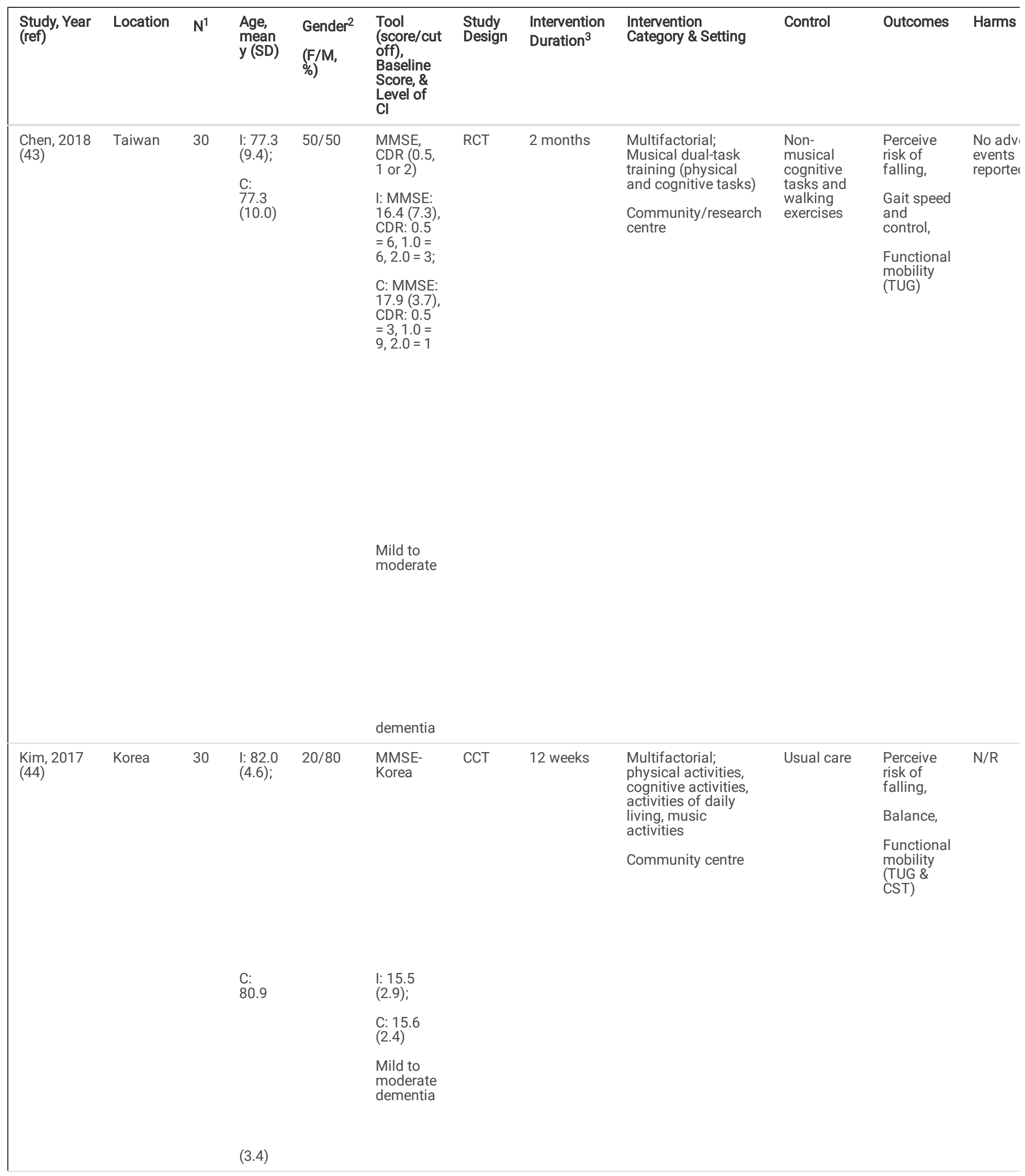

$\mathrm{Cl}=$ cognitive impairment; $\mathrm{O}=$ overall population; $\mathrm{I}=$ intervention; $\mathrm{C}=$ control; $\mathrm{AD}=$ Alzheimer's Disease; $\mathrm{N} / \mathrm{R}=$ not reported; $\mathrm{RCT}=$ randomized controlled trial; $\mathrm{C}$ $=$ clinical (non-randomized) controlled trial. MoCA = Montreal cognitive assessment (Score /30); MMSE = Mini Mental State Exam; ACE-R = Addenbrooke's cognitive examination - revised; $C D R=$ Clinical Dementia Rating scale; TUG = timed up and go test; $C S T$ = chair sit stand test; $F R T$ = functional reach test 


\begin{tabular}{|c|c|c|c|c|c|c|c|c|c|c|c|}
\hline $\begin{array}{l}\text { Study, Year } \\
\text { (ref) }\end{array}$ & Location & $\mathbf{N}^{1}$ & $\begin{array}{l}\text { Age, } \\
\text { mean } \\
\text { y (SD) }\end{array}$ & $\begin{array}{l}\text { Gender }^{2} \\
(\mathrm{~F} / \mathrm{M}, \\
\%)\end{array}$ & $\begin{array}{l}\text { Tool } \\
\text { (score/cut } \\
\text { off), } \\
\text { Baseline } \\
\text { Score, \& } \\
\text { Level of } \\
\text { Cl }\end{array}$ & $\begin{array}{l}\text { Study } \\
\text { Design }\end{array}$ & $\begin{array}{l}\text { Intervention } \\
\text { Duration }\end{array}$ & $\begin{array}{l}\text { Intervention } \\
\text { Category \& Setting }\end{array}$ & Control & Outcomes & Harms \\
\hline $\begin{array}{l}\text { Wesson, } \\
2013 \text { (10) }\end{array}$ & Australia & 22 & $\begin{array}{l}\text { I: } 78.7 \\
(4.2) \\
\text { C: } \\
80.9 \\
(5.0)\end{array}$ & $41 / 59$ & $\begin{array}{l}\text { ACE-R ( } \leq \\
82) \text {, } \\
\text { MMSE } \\
\text { I: ACE-R: } \\
67.8 \\
(12.6) \text {, } \\
\text { MMSE: } \\
24.5(3.1) \text {; } \\
\text { C: ACE-R: } \\
62.5 \\
(14.2) \text {, } \\
\text { MMSE: } \\
22.5 \text { (4.3) } \\
\text { Mild } \\
\text { dementia }\end{array}$ & RCT & 12 weeks & $\begin{array}{l}\text { Multifactorial; } \\
\text { strength and balance } \\
\text { exercises, home } \\
\text { hazard reduction } \\
\text { Home-based }\end{array}$ & $\begin{array}{l}\text { Usual care, } \\
\text { health } \\
\text { promotion } \\
\text { brochures } \\
\text { on fall } \\
\text { prevention } \\
\text { and home } \\
\text { safety }\end{array}$ & $\begin{array}{l}\text { Falls, } \\
\text { Perceive } \\
\text { risk of } \\
\text { falling, } \\
\text { Balance, } \\
\text { Gait speed } \\
\text { and } \\
\text { control }\end{array}$ & $\begin{array}{l}\text { No seriı } \\
\text { advers } \\
\text { events } \\
\text { related } \\
\text { the } \\
\text { interver } \\
\text { were } \\
\text { reporteı } \\
\text { Minor } \\
\text { compla } \\
\text { relating } \\
\text { stiffnes } \\
\text { dizzine } \\
\text { and mil } \\
\text { joint pa } \\
\text { (n= 4; } 3 \\
\text { were } \\
\text { reporteı }\end{array}$ \\
\hline $\begin{array}{l}\text { Suttanon, } \\
2013(41)\end{array}$ & Australia & 40 & $\begin{array}{l}\text { O: } \\
81.90 \\
(5.72) \text {; } \\
\text { l: } \\
83.42 \\
(5.10) \text {; } \\
\text { C: } \\
80.52 \\
(6.01)\end{array}$ & $63 / 37$ & $\begin{array}{l}\text { MMSE ( } \geq \\
10) \\
\text { I: } 20.89 \\
(4.74) ; \\
\text { C: } 21.67 \\
(4.43) \\
\text { Mild to } \\
\text { moderate } \\
\text { AD }\end{array}$ & RCT & 6 months & $\begin{array}{l}\text { Exercise; balance } \\
\text { and strength } \\
\text { exercises, walking } \\
\text { program } \\
\text { Home-based }\end{array}$ & $\begin{array}{l}\text { Education } \\
\text { and } \\
\text { information } \\
\text { sessions on } \\
\text { the topic of } \\
\text { dementia } \\
\text { and ageing }\end{array}$ & $\begin{array}{l}\text { Falls, } \\
\text { Perceive } \\
\text { risk of } \\
\text { falling, } \\
\text { Balance, } \\
\text { Gait speed } \\
\text { and } \\
\text { control, } \\
\text { Functional } \\
\text { mobility } \\
(\text { TUG, CST, } \\
\text { and FRT*) }\end{array}$ & $\begin{array}{l}\text { There n } \\
\text { no falls } \\
\text { other } \\
\text { serious } \\
\text { adverse } \\
\text { events } \\
\text { associc } \\
\text { with the } \\
\text { interver }\end{array}$ \\
\hline $\begin{array}{l}\text { Hernandez, } \\
2010 \text { (42) }\end{array}$ & Brazil & 20 & $\begin{array}{l}\text { O: } \\
78.5 \\
(6.8) ; \\
\text { I: } 77.7 \\
(7.6) ; \\
\text { C: } \\
84.0 \\
(6.1)\end{array}$ & N/R & $\begin{array}{l}\text { CDR; } \\
\text { MMSE } \\
\text { I: } 16.4 \\
(6.7) ; \\
\text { C: } 14.2 \\
(5.1) \\
\text { Mild to } \\
\text { moderate } \\
\text { AD }\end{array}$ & ССТ & 6 months & $\begin{array}{l}\text { Exercise; stretching, } \\
\text { weight training, } \\
\text { circuits, dance, } \\
\text { recreational } \\
\text { activities, relaxation } \\
\text { N/R }\end{array}$ & Usual care & $\begin{array}{l}\text { Balance, } \\
\text { Functional } \\
\text { mobility } \\
\text { (TUG) }\end{array}$ & $\mathrm{N} / \mathrm{R}$ \\
\hline
\end{tabular}

$\mathrm{Cl}=$ cognitive impairment; $\mathrm{O}=$ overall population; $\mathrm{I}=$ intervention; $\mathrm{C}=$ control; $\mathrm{AD}=$ Alzheimer's Disease; $\mathrm{N} / \mathrm{R}=$ not reported; $\mathrm{RCT}=$ randomized controlled trial; $\mathrm{C}$ = clinical (non-randomized) controlled trial. MoCA = Montreal cognitive assessment (Score /30); MMSE = Mini Mental State Exam; ACE-R = Addenbrooke's cognitive examination - revised; $C D R=$ Clinical Dementia Rating scale; TUG = timed up and go test; $C S T$ = chair sit stand test; FRT = functional reach test

1. Number of participants randomized to intervention; 2 . Values for gender are based on reported baseline which may not equal $\mathrm{N}$ randomized but rather the number of participants who completed the intervention; 3 . Not including follow-up, if applicable; * = outcome not meta-analyzed

\section{Risk of Bias and Quality of Included Studies}

The Cochrane RoB tool showed mixed quality of study methodology: 2 studies were low risk of bias (37, 39), 3 studies were high risk of bias (40, 42,43$)$, and 7 studies were rated as an unclear risk of bias $(10,35,36,38,41,44,45)$, mostly due to issues around allocation concealment, blinding, and incomplete outcome data (Table 2).

Table 2. Risk of Bias for Included Studies 


\begin{tabular}{|c|c|c|c|c|c|c|}
\hline Author & $\begin{array}{l}\text { SEQUENCE } \\
\text { GENERATION }\end{array}$ & $\begin{array}{l}\text { ALLOCATION } \\
\text { CONCEALMENT }\end{array}$ & $\begin{array}{l}\text { BUINDING OF OUTCOME } \\
\text { ASSESSMENT }\end{array}$ & $\begin{array}{l}\text { INCOMPLETE } \\
\text { OUTCOME DATA }\end{array}$ & $\begin{array}{l}\text { SELECTIVE } \\
\text { REPORTING }\end{array}$ & \begin{tabular}{|l|} 
OTHER \\
BIAS \\
\end{tabular} \\
\hline Varriano, $2020(35)$ & $\mathrm{L}$ & $u$ & U & $\mathrm{H}$ & $\mathrm{U}$ & $\mathrm{U}$ \\
\hline Goldberg, 2019 (36) & $\mathbf{L}$ & $\mathrm{H}$ & $\mathrm{H}$ & $\mathrm{L}$ & $\mathrm{L}$ & $\mathrm{L}$ \\
\hline Padala, 2017 (37) & L & $\mathrm{L}$ & $\mathrm{H}$ & $\mathrm{L}$ & L & $\mathrm{L}$ \\
\hline Zieschang, 2017 (38) & $u$ & $u$ & U & $\mathrm{L}$ & $\mathrm{L}$ & L \\
\hline Sungkarat, 2017 (39) & $\mathrm{L}$ & $\mathrm{L}$ & $\mathrm{H}$ & $\mathrm{L}$ & $\mathrm{L}$ & $\underline{L}$ \\
\hline Schwenk, 2016 (40) & L & $\mathrm{H}$ & $\mathrm{H}$ & $\mathrm{L}$ & $\mathrm{L}$ & $\mathrm{U}$ \\
\hline $\begin{array}{l}\text { Montero-Odasso, } 2019 \\
(45)\end{array}$ & $\mathrm{L}$ & $\mathrm{L}$ & $\mathrm{L}$ & $\mathrm{H}$ & $\mathrm{L}$ & $\mathrm{H}$ \\
\hline Chen, 2018 (43) & $\mathrm{L}$ & $\mathrm{H}$ & $\mathrm{H}$ & $\mathrm{H}$ & $\mathrm{L}$ & $\mathrm{H}$ \\
\hline Kim, 2017 (44) & $u$ & $u$ & $u$ & U & $\mathrm{U}$ & $\mathrm{H}$ \\
\hline Wesson, $2013(10)$ & $\mathrm{L}$ & $\mathrm{L}$ & $\mathrm{H}$ & $\mathrm{H}$ & $\mathrm{L}$ & $\mathrm{L}$ \\
\hline Suttanon, 2013 (41) & $\mathrm{L}$ & $\mathrm{L}$ & $\mathrm{H}$ & $\mathrm{H}$ & $\mathrm{L}$ & $\mathrm{L}$ \\
\hline Hernandez, 2010 (42) & $\mathrm{H}$ & $u$ & U & $\mathrm{H}$ & L & $\mathrm{U}$ \\
\hline
\end{tabular}

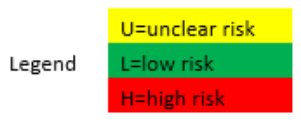

The certainty of evidence, as assessed by GRADE, ranged from very low to moderate but was moderate for most outcomes ( $n=4$ ) due to downgrading for risk of bias concerns (Table 3; full GRADE tables by outcome can be found in Additional File 4).

Table 3

Benefits of Treatment; Results of Meta-Analysis by Outcome for Included Studies ( $n=12)$

\begin{tabular}{|c|c|c|c|}
\hline Outcome & \# studies | N & SMD ( $95 \%$ Confidence interval) & GRADE rating \\
\hline \multirow[t]{2}{*}{ Falls (\# events) } & $4 \mid 224$ & RR $0.99(0.60,1.65)$ & LOW \\
\hline & & & downgraded for risk of bias and imprecision \\
\hline \multirow[t]{2}{*}{ Falls (incidence) } & $4 \mid 209$ & IR $0.90(0.47,1.71)$ & LOW \\
\hline & & & downgraded for risk of bias and imprecision \\
\hline \multirow[t]{2}{*}{ Perceived Risk of Falls / Fear of Falling } & $8 \mid 263$ & Medium; $-0.73(-1.10,-0.36)$ & MODERATE \\
\hline & & & downgraded for risk of bias \\
\hline \multirow[t]{2}{*}{ Balance } & $9 \mid 318$ & Medium; $0.66(0.19,1.12)$ & MODERATE \\
\hline & & & downgraded for risk of bias \\
\hline \multirow[t]{2}{*}{ Gait speed and control } & $6 \mid 194$ & Small; $0.26(0.08,0.43)$ & MODERATE \\
\hline & & & downgraded for risk of bias \\
\hline \multirow[t]{2}{*}{ TUG } & $5 \mid 151$ & Medium; $-0.56(-0.94,-0.17)$ & MODERATE \\
\hline & & & downgraded for risk of bias \\
\hline \multirow[t]{2}{*}{ CST } & $2 \mid 70$ & No effect; $0.34(-1.73,1.06)$ & VERY LOW \\
\hline & & & downgraded for risk of bias and imprecision \\
\hline
\end{tabular}

Bold denotes significance $\mathrm{p}<0.05 ; \mathrm{N}=$ total number of participants; $\mathrm{SMD}=$ standardized mean difference; $\mathrm{RR}=$ risk ratio; $\mathrm{IR}=$ incidence rate; $\mathrm{TUG}=$ timed up and go test; CST = chair sit and stand test

\section{Benefits of Treatment}

We conducted a meta-analysis for all outcomes on 11 of the included studies. One study (35) was excluded from the meta-analysis and is only described qualitatively because of severe risk of bias concerns and the loss of all participants but 1 in each group during follow-up. Additionally, of the potential functional mobility outcome measures, our included studies only had data for the Chair Sit and Stand Test (CST) and the Timed Up and Go Test (TUG). Therefore, we analyzed these measures separately.

A number of different measures were used across the included studies for the various outcomes (Additional File 5). Falls as a direct outcome was measured by 5 different studies as numbers of falls (events) $(n=4)$ or incidence of falls (time to event) $(n=4)$ using self-reported falls calendars. Perceived risk of falls or fear of falls was measured by 8 studies using the Falls Efficacy Scale by 6 of the studies with this outcome. Other common measures included the Falls Risk for Older People or Physiological Profile Assessment. Balance was the most frequently reported outcome as it was measured by 9 different studies and included measurements such as the Berg Balance Scale, Postural Sway Tests, and Limits of Stability. Gait speed and control were reported by 6 studies and included a variety of gait speed measurements (habitual walking speed, fast walking speed), step tests and walk tests, Dual-task gait cost tests, and gait 
coefficient of variation. Lastly, functional mobility outcomes were assessed by either TUG and/or CST. Five studies assessed TUG with some variations in this test such as including cognitive or motor tasks. Only 2 studies assessed CST or sit to stand measurements.

Overall, fall prevention interventions had significant effects of medium magnitude on perceived risk of falls/fear of falling (SMD - 0.73 [-1.10, -0.36$]$ ), balance (SMD $0.66[0.19,1.12])$, and functional mobility as measured by TUG (SMD - $0.56[-0.94,-0.17])$ and significant effects of small magnitude on gait speed and control (SMD $0.26[0.08,0.43]$ ) all with moderate certainty of evidence (Fig. 2; Table 3). There were no significant effects for falls (number of events or falls incidence) and chair sit stand test outcomes (Table 3).

We were able to conduct subgroup analysis by intervention type and risk of bias for perceived risk of falls/fear of falling, balance, and gait speed and control as these outcomes had more than 5 studies with data (Table 4; Additional File 6). Subgroup analysis for balance showed that the benefit was significant for exercise interventions (SMD $0.85[0.26,1.43]$ ) and low risk of bias studies (SMD $1.28[0.20,2.36]$ ); however, the difference between intervention subgroups or risk of bias subgroups was not statistically significant $(p=0.44$ and $p=0.35$, respectively). Perceived risk of falls was measured in two intervention types (exercise and multifactorial) and subgroup analysis showed that the benefit was again significant for exercise interventions (SMD - 0.70 [-1.02, -0.32]). The benefit was also significant for low (SMD - $0.88[-1.67,-0.08])$ and unclear (SMD - $0.77[-1.32,-0.22)]$ risk of bias studies. However, for all subgroup analysis, the difference between subgroups was not statistically significant with $p=0.93$ for intervention type and $p=0.84$ for risk of bias. Lastly, subgroup analysis for gait control and stability shows that the benefit was significant for medication interventions (SMD $0.42[0.09,0.75$ )] and unclear risk of bias studies (SMD 0.27 $[0.03,0.51])$, although there was only one medication intervention that measured gait speed and control and there was no low risk of bias studies. Again, the difference between subgroups was not statistically significant with $p=0.27$ for intervention type and $p=0.75$ for risk of bias. 
Table 4

Results of Subgroup Analysis

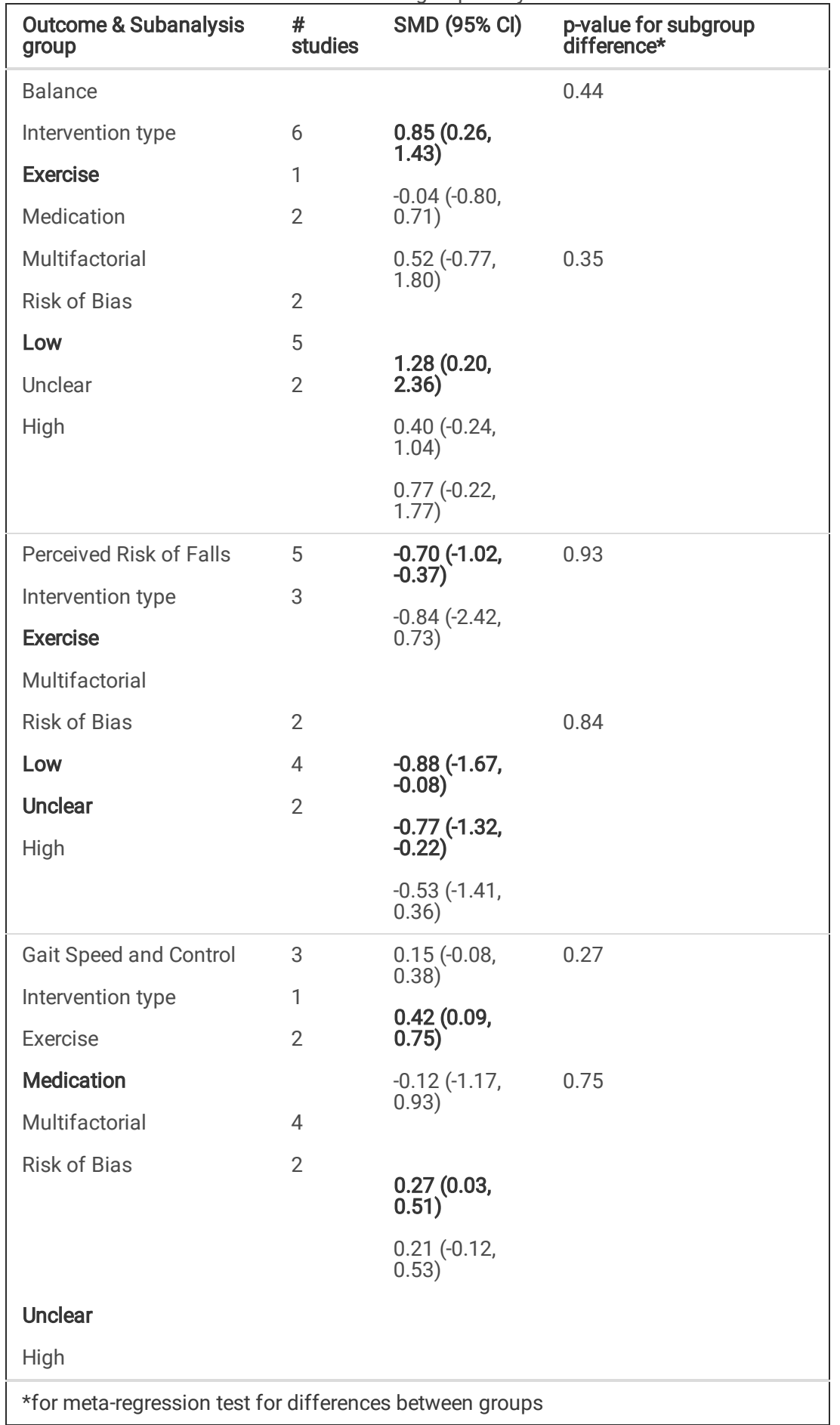

BOLD denotes summary subgroup estimates that are statistically significant with $p<0.05$

$\mathrm{SMD}=$ standardized mean difference, $\mathrm{Cl}=$ confidence interval

\section{Discussion}

The purpose of this review was to evaluate the available evidence on the effectiveness of primary and secondary fall prevention interventions in communitydwelling adults with mild to moderate cognitive impairment. The effectiveness of the 12 included RCTs was assessed based on six outcomes which were ranked by our interdisciplinary research team and included falls, perceived risk of falls/fear or concern of falls, balance, gait speed and control, and the two functional mobility outcomes of TUG and CST. The meta-analysis demonstrated that fall prevention interventions could be effective for decreasing participants' perceived risk of falls/fear of falling, and improving their balance, gait speed and control, and TUG based on moderate certainty of evidence. But there were no significant effects for falls (number of events or falls incidence) and chair sit stand test outcomes. The subgroup analysis of our review also demonstrated that exercise interventions and high quality studies at lower risk of bias maintained these benefits, but not significantly more than other 
interventions or studies at a higher risk of bias. However, caution should be taken when interpreting the findings of our systematic review due to the small number of studies, small sample sizes in the included studies, and the heterogeneity of interventions and participants in the studies.

\section{Comparison with Existing Literature}

While the body of evidence for fall prevention continues to grow, there are limited studies and reviews which include meta-analyses on interventions to specifically help adults with cognitive impairment in a community-based setting, despite these people having a disproportionately high rate of falls and fractures and poorer outcomes after falls $(10,11)$. Published reviews are either focused on a general older adult population $(12,48)$, on specific intervention types such as physical activity $(13,14,49)$, or have focused on more mixed populations (including many types of $\mathrm{Cl})$ and settings $(3,9,15)$.

Cognitive impairment is a well-recognized risk factor for falls due to a variety of reasons including a lack of insight into environmental dangers or tripping hazards, a failure to comply with safety or medical treatment, an increased state of confusion, and the role cognition plays in gait $(3,50)$. However, a better understanding is required of fall risk factors in this population (50). In cognitively healthy adults and older adults, there are over 400 risk factors associated with falls (4). Our review chose to include numerous outcomes and measurements/tools used to assess these outcomes to allow for a robust body of evidence and assessment of the effectiveness of fall prevention interventions in this population. The outcomes which showed significance from our metaanalysis of included fall prevention studies aligns with short-form screening tools such as the Stopping Elderly Accidents, Deaths, and Injuries (STEADI) initiative and the associated 3-item fall screening questionnaire (51). This brief screening tool simply asks if the individual has fallen in the past year (falls outcome), feels unsteady when standing or walking (balance and gait outcomes), and if they are worried about falling (perceived fear of falls outcome). While there were no significant improvements in falls in our review, the surrogate outcomes which are related to falls risk provide signals and good data that there could be improvements in falls themselves. It is encouraging to see trends in four outcomes from moderate certainty of evidence. However, it is important to note that these outcomes have varying reliability in individuals with cognitive impairment (52-55) and the falls outcome was downgraded for certainty of evidence because of methodological issues and concerns related to risk of bias and imprecision.

The downgrading of evidence speaks to another important finding with implications for future research in this area related to study design and study quality. The subgroup analysis of this review signalled that higher quality studies showed beneficial and significant effects in outcomes related to fall prevention including balance, perceived risk of falls, and gait control and stability. These results must still be interpreted with caution due to the limited number of studies in our review overall and within each subgroup (as is evident by the wide confidence intervals), the possibility for underpowered studies with small sample sizes, and the high degree of heterogeneity across these interventions (as is evident by the variety of exercise interventions). Many of the included studies did not indicate that falls was their primary outcome and overall, the interventions were very short in duration, which may mean these studies were not equipped to see significant effects in outcomes which take longer to change. However, it is promising that the subgroup analysis showed consistent findings and shows the need for more robust, high quality research to ascertain these findings.

Another criticism of previous fall prevention reviews in those with cognitive impairment is the heterogeneity of sample population (3) and the same is true in our review. While we had strict definitions and criteria for inclusion of studies based on population, we found a broad range of participants in terms of how they were classified and diagnosed and the tools and cut-off scores that were used to characterize mild and moderate cognitive impairment. The diversity in the included participants of the studies from this review may have also made it difficult to see significant changes in the outcomes of interest as clinicians have stated the importance of appropriate timing for intervening with this population based on their cognitive abilities. This heterogeneity also limited our ability to conduct any subgroup analysis according to cognitive level.

Exercise and multifactorial interventions are the most frequently reported fall prevention studies in those both with and without cognitive impairment (3). Similar to previous reviews $(3,14)$, we also found that exercise interventions were effective at showing benefit for outcomes related to fall risk factors in individuals with cognitive impairment. However, the evidence was less convincing for multifactorial interventions and neither intervention type was statistically effective at reducing or preventing falls themselves nor were exercise interventions statistically better than multifactorial interventions. While these findings are similar to the results of reviews in adults without cognitive impairment (48), it speaks to the challenges and complex factors that affect factors related to falls and the uniqueness of a population with cognitive impairment. There may need to be intervention modifications for cognitively impaired adults such as including the engagement of carers, regular contact by trained professionals, a greater choice of exercise, among others $(10,41)$. We also were unable to do any subgroup analysis or investigation to further elicit the intensities, duration, components, or combination of these factors which may be related to their effectiveness.

\section{Limitations}

Although our search was comprehensive, we did not explicitly search grey literature. We did verify our included studies with those of other similar reviews and our results align with previous research in this area (13-15). While we were consistent in our application of our inclusion criteria for mild to moderate cognitive impairment, we recognize that a limitation of this review will be how authors report and describe their study participant's level of cognitive impairment. There was ambiguity in how authors defined and used cut offs, which speaks to issues within this field of study and the limitations of using screening tests for study purposes in the absence of a true clinical diagnosis. Lastly, while we did not observe any significant asymmetry across funnel plots for publication bias, studies were and have risk of bias concerns.

\section{Implications for Practice and Research}

The findings from our review make it difficult to make clear recommendations for practice. Research in falls prevention among community-dwelling adults is still largely conducted on individuals without cognitive impairment and our review shows the difficulties in translating this research into practice in patients with cognitive impairment. The greater fall risk for adults with cognitive impairment includes different underlying mechanisms and there may be unique risk factors that are not present in cognitively normal adults. The heterogeneity of study participants and intervention components does not help explain the

Page 12/18 
findings or suggest best practices for clinical practice. Clinicians should continue to work with their patients to implement fall prevention activities such as exercise and look for improvements in fall risk factors such as perceived risk of falls, balance, gait, and TUG.

Our review also demonstrates that evidence is still lacking for fall prevention interventions in those with cognitive impairment and further highlights that the needs of clinicians are not being met, despite calls advocating for large-scale, high quality research (3). We found small studies, that were short in duration, not powered to detect falls, and had risk of bias issues. Subgroup analysis indicated that we need more high quality research (low risk of bias studies) to ascertain the findings from our review.

\section{Conclusions}

Practitioners frequently see exercise interventions in recommendations for fall prevention but are unsure what they can do to help their patients with cognitive impairment. Our review and meta-analysis confirm the difficulties and limitations in this field of research, which echoes what practitioners are saying. However, there is promising evidence which suggests that fall prevention interventions, particularly exercise programs of all kinds, can assist in the improvement of fall risk factors in this population. Further high quality research is needed with longer durations and appropriately powered sample sizes to determine the most appropriate falls prevention interventions for community-dwelling adults with cognitive impairment.

\section{Abbreviations}

\section{ACE-R}

Addenbrooke's cognitive examination - revised

$A D$

Alzheimer's Disease

ADLs

activities of daily living

C

control

CCT

clinical controlled trial

CDR

clinical dementia rating scale

CENTRAL

Cochrane Central Register of Controlled Trials

\section{$\mathrm{Cl}$}

cognitive impairment

CINAHL

Cumulative Index of Nursing and Allied Health Literature

CoV

coefficient of variation

CST

chair sit stand test

DGI

dynamic gait index

DSM-IV

Diagnostic and Statistical Manual of Mental Disorders

DTC

dual-task cost

FES

falls efficacy scale

FROp

Falls risk for older people

FRT

functional reach test

GRADE

Grading of Recommendation, Assessment, Development and Evaluations

I

intervention

ICONFES

Iconographical falls efficacy scale

IR

incidence rate

IV 
inverse variance

$\mathrm{MCl}$

mild cognitive impairment

MD

mean difference

MMSE

Mini Mental State Exam

MoCA

Montreal Cognitive Assessment

N

total number of participants

$\mathrm{N} / \mathrm{R}$

not reported

0

overall population

PPA

Physiological profile assessment

PRISMA

Preferred Reporting Items for Systematic Reviews and Meta-analyses

RCT

randomized controlled trial

RoB

risk of bias

RR

risk ratio

SMD

standardized mean difference

STEADI

Stopping Elderly Accidents, Deaths, and Injuries

TUG

timed up and go test

$6 \mathrm{MWT}$

6 metre walk test

\section{Declarations}

Ethics approval

As this study was solely literature based and did not involve any research partiipants or subjects, no formal ethics approval from McMaster Research Ethics Board was required.

\section{Consent for publication}

Not Applicable

\section{Availability of data and materials}

The main study data is the data extraction materials and quality ratings of included papers, most of which are included in the manuscript tables. Any other supporting data relating to this review is available from the authors.

\section{Competing interests}

The authors declare that they have no competing interests.

\section{Funding}

This research was funded by the Ontario Neurotrauma Foundation (ONF), the McMaster Evidence Review and Synthesis Team (MERST), and the Aging, Community and Health Research Unit (ACHRU) at McMaster University. ONF is funded by the Ontario Ministry of Health. ACHRU is funded by the Max Bell Foundation, Ontario SPOR Support Unit (OSSU), Canadian Institutes of Health Research (CIHR): Catalyst Fund and Pan-Canadian SPOR Network in Primary 
and Integrated Health Care Innovations (PIHCl), Labarge Optimal Aging Initiative, Labarge Foundation, McMaster Institute for Research on Aging (MIRA), and Diabetes Action Canada. Together, these funds supported the work of Dr. Megan Racey as a post-doctoral fellow. Dr. Diana Sherifali holds the Heather M Arthur Population Health Research Institute/Hamilton Health Sciences Chair in Interprofessional Health Research which supported her role in this work. The funders approved the protocol of the study and the final manuscript, but had no role in the screening, data extraction, or data analysis.

\section{Authors' contributions}

All authors were involved in conception and design of the study and approved the protocol; MR, DFL, DS were responsible for overseeing the search of databases and literature and $\mathrm{RL}$ handled management of database and deduplication or records. MR, DFL, RL, MJ were involved in the screening of citations; MR, DFL, MJ were responsible for data extraction; MR, DFL, MUA, DS were responsible for data verification and analysis of data. MR, DFL, MUA, HG, SH, JP, RS, LH, DS were involved in interpretation of data. All authors supported in the drafting of the manuscript which was led by MR and all authors supported in revising and formatting of the manuscript. All authors have provided final approval of the version of the manuscript submitted for publication, and all authors agree to be accountable for all aspects of the work.

\section{Acknowledgements}

The authors thank Angela Eady for developing the search strategy.

\section{References}

1. Allali G, Launay CP, Blumen HM, Callisaya ML, De Cock AM, Kressig RW, et al. Falls, Cognitive Impairment, and Gait Performance: Results From the GOOD Initiative. Journal of the American Medical Directors Association. 2017;18(4):335-40.

2. Canada PHAo. Seniors' Falls in Canada: Second Report. 2014.

3. Booth V, Logan P, Harwood R, Hood V. Falls prevention interventions in older adults with cognitive impairment: A systematic review of reviews. International Journal of Therapy \& Rehabilitation. 2015;22(6):289-96.

4. Markle-Reid M, Browne G, Gafni A, Roberts J, Weir R, Thabane L, et al. A cross-sectional study of the prevalence, correlates, and costs of falls in older home care clients 'at risk' for falling. Can J Aging. 2010;29(1):119-37.

5. Fernando E, Fraser M, Hendriksen J, Kim CH, Muir-Hunter SW. Risk Factors Associated with Falls in Older Adults with Dementia: A Systematic Review. Physiother Can. 2017;69(2):161-70.

6. Beauchet O, Sekhon H, Schott A-M, Rolland Y, Muir-Hunter S, Markle-Reid M, et al. Motoric Cognitive Risk Syndrome and Risk for Falls, Their Recurrence, and Postfall Fractures: Results From a Prospective Observational Population-Based Cohort Study. Journal of the American Medical Directors Association. 2019;20(10):1268-73.

7. Nasreddine ZS, Phillips NA, Bédirian V, Charbonneau S, Whitehead V, Collin I, et al. The Montreal Cognitive Assessment, MoCA: A Brief Screening Tool For Mild Cognitive Impairment. Journal of the American Geriatrics Society. 2005;53(4):695-9.

8. Winblad Bea. Mild cognitive impairment - beyond controversies, towards

9. a consensus: report of the International Working Group

10. on Mild Cognitive Impairment. Journal of Internal Medicine. 2004;256(3):240-6.

11. Sherrington C, Michaleff ZA, Fairhall N, Paul SS, Tiedemann A, Whitney J, et al. Exercise to prevent falls in older adults: an updated systematic review and meta-analysis. British journal of sports medicine. 2017;51(24):1750-8.

12. Wesson J, Clemson L, Brodaty H, Lord S, Taylor M, Gitlin L, et al. A feasibility study and pilot randomised trial of a tailored prevention program to reduce falls in older people with mild dementia. BMC geriatrics. 2013;13:89.

13. Winter $\mathrm{H}$, Watt $\mathrm{K}$, Peel NM. Falls prevention interventions for community-dwelling older persons with cognitive impairment: A systematic review. International Psychogeriatrics. 2013;25(2):215-27.

14. Hopewell S, Copsey B, Nicolson P, Adedire B, Boniface G, Lamb S. Multifactorial interventions for preventing falls in older people living in the community: a systematic review and meta-analysis of 41 trials and almost 20000 participants. Br J Sports Med. 2020;54(22):1340-50.

15. Lipardo DS, Aseron AMC, Kwan MM, Tsang WW. Effect of Exercise and Cognitive Training on Falls and Fall-Related Factors in Older Adults With Mild Cognitive Impairment: A Systematic Review. Archives of Physical Medicine \& Rehabilitation. 2017;98(10):2079-96.

16. Burton E, Cavalheri V, Adams R, Browne CO, Bovery-Spencer P, Fenton AM, et al. Effectiveness of exercise programs to reduce falls in older people with dementia living in the community: a systematic review and meta-analysis. Clin Interv Aging. 2015;10:421-34.

17. Guo JL, Tsai YY, Liao JY, Tu HM, Huang CM. Interventions to reduce the number of falls among older adults with/without cognitive impairment: An exploratory meta-analysis. International Journal of Geriatric Psychiatry. 2014;29(7):661-9.

18. Foundation ON. Falls Prevention Community of Practice LOOP [Available from: https://www.fallsloop.com/. 
19. Panel on Prevention of Falls in Older Persons AGS, British Geriatrics S. Summary of the Updated American Geriatrics Society/British Geriatrics Society clinical practice guideline for prevention of falls in older persons. J Am Geriatr Soc. 2011;59(1):148-57.

20. Moher D, Liberati A, Tetzlaff J, Altman DG, Group P. Preferred reporting items for systematic reviews and meta-analyses: the PRISMA statement. J Clin Epidemiol. 2009;62(10):1006-12.

21. Kim H, Awata S, Watanabe Y, Kojima N, Osuka Y, Motokawa K, et al. Cognitive frailty in community-dwelling older Japanese people: Prevalence and its association with falls. Geriatr Gerontol Int. 2019;13:13.

22. Institute for Work and Health. Primary, secondary and tertiary prevention Toronto: IWH; 2015 [Available from: https://www.iwh.on.ca/what-researchersmean-by/primary-secondary-and-tertiary-prevention.

23. Guyatt GH, Oxman AD, Kunz R, Atkins D, Brozek J, Vist G, et al. GRADE guidelines: 2. Framing the question and deciding on important outcomes. Journal of Clinical Epidemiology. 2011;64(4):395-400.

24. Markle-Reid MF, Dykeman CS, Reimer HD, Boratto LJ, Goodall CE, McGugan JL. Engaging community organizations in falls prevention for older adults: Moving from research to action. Canadian journal of public health = Revue canadienne de sante publique. 2015;106(4):e189-96.

25. Lamb SE, Becker C, Gillespie LD, Smith JL, Finnegan S, Potter R, et al. Reporting of complex interventions in clinical trials: development of a taxonomy to classify and describe fall-prevention interventions. Trials. 2011;12(1):125

26. Higgins JP, Altman DG, Gotzsche PC, Juni P, Moher D, Oxman AD, et al. The Cochrane Collaboration's tool for assessing risk of bias in randomised trials. BMJ. 2011;343:d5928.

27. Schünemann H BJ, Guyatt G, Oxman A, editors. GRADE handbook for grading quality of evidence and strength of recommendations. Updated October 2013. The GRADE Working Group. 2013.

28. GDT G. GRADEpro Guideline Development Tool [Software]. McMaster University. 2015.

29. DerSimonian R Fau - Laird N, Laird N. Meta-analysis in clinical trials. Control Clin Trials. 1986;7(3):177-88.

30. Higgins JPT LT, Deeks JJ. Choosing effect measures and computing estimates of effect. In: Higgins JPT LT, Deeks JJ, editor. Cochrane Handbook for Systematic Reviews of Interventions. version 6.1 ed2019. p. 143 - 76.

31. Assink M, Wibbelink CJM. Fitting three-level meta-analytic models in R: A step-by-step tutorial. The Quantitative Methods for Psychology. 2016;12(3):15474.

32. Cohen J. Statistical Power Analysis for the Behavioral Sciences (2nd ed.). Hillsdale, NJ: Lawrence Erlbaum Associates, Publishers; 1988.

33. Pastor DA, Lazowski RA. On the Multilevel Nature of Meta-Analysis: A Tutorial, Comparison of Software Programs, and Discussion of Analytic Choices. Multivariate Behav Res. 2018;53(1):74-89.

34. Pastor DA, Lazowski RA. On the Multilevel Nature of Meta-Analysis: A Tutorial, Comparison of Software Programs, and Discussion of Analytic Choices. Multivariate Behavioral Research. 2018;53(1):74-89.

35. Viechtbauer W. Conducting Meta-Analyses in R with the metafor Package. Journal of Statistical Software. 2010;36(3).

36. Harrer M CP, Furukawa T, Ebert DD dmetar: Companion R Package For The Guide 'Doing Meta-Analysis in R'. R package version 0.0.9000. 2019.

37. Varriano B, Sulway S, Wetmore C, Dillon W, Misquitta K, Multani N, et al. Vestibular Exercises as a Fall Prevention Strategy in Patients with Cognitive Impairment. Canadian Journal of Neurological Sciences. 2020;47(1):126-30.

38. Goldberg SE, van der Wardt V, Brand A, Burgon C, Bajwa R, Hoare Z, et al. Promoting activity, Independence and stability in early dementia (PrAISED): a, multisite, randomised controlled, feasibility trial. BMC Geriatrics. 2019;19(1):1-12.

39. Padala KP, Padala P, Lensing SY, Dennis RA, Bopp MM, Parkes CM, et al. Home-based Exercise Program Improves Balance and Fear of Falling in Community Dwelling Older Adults with Mild Alzheimer's Disease. Journal of the American Geriatrics Society. 2017;65:S9-S10.

40. Zieschang T, Schwenk M, Becker C, Uhlmann L, Oster P, Hauer K. Falls and Physical Activity in Persons with Mild to Moderate Dementia Participating in an Intensive Motor Training Randomized Controlled Trial. Alzheimer Disease and Associated Disorders. 2017;31(4):307-14.

41. Sungkarat S, Boripuntakul S, Chattipakorn N, Watcharasaksilp K, Lord SR. Effects of Tai Chi on Cognition and Fall Risk in Older Adults with Mild Cognitive Impairment: A Randomized Controlled Trial. Journal of the American Geriatrics Society. 2017;65(4):721-7.

42. Schwenk M, Sabbagh M, Lin I, Morgan P, Grewal GS, Mohler J, et al. Sensor-based balance training with motion feedback in people with mild cognitive impairment. Journal of Rehabilitation Research and Development. 2016;53(6):945-58.

43. Suttanon P, Hill KD, Said CM, Williams SB, Byrne KN, LoGiudice D, et al. Feasibility, safety and preliminary evidence of the effectiveness of a home-based exercise programme for older people with Alzheimer's disease: a pilot randomized controlled trial. Clinical rehabilitation. 2013;27(5):427-38.

44. Hernandez SSS, Coelho FGM, Gobbi S, Stella F. Effects of physical activity on cognitive functions, balance and risk of falls in elderly patients with Alzheimer's dementia. Brazilian Journal of Physical Therapy. 2010;14(1):68-74.

45. Chen YL, Pei YC. Musical dual-task training in patients with mild-to-moderate dementia: A randomized controlled trial. Neuropsychiatric Disease and Treatment. 2018;14:1381-93.

46. Kim KU, Kim SH, Oh HW. The effects of occupation-centered activity program on fall-related factors and quality of life in patients with dementia. J Phys Ther Sci. 2017;29(7):1188-91.

47. Montero-Odasso M, Speechley M, Chertkow H, Sarquis-Adamson $Y$, Wells J, Borrie M, et al. Donepezil for gait and falls in mild cognitive impairment: A randomized controlled trial. European Journal of Neurology. 2019;26(4):651-9.

48. Petersen RC, Smith GE, Waring SC, Ivnik RJ, Kokmen E, Tangelos EG. Aging, memory, and mild cognitive impairment. Int Psychogeriatr. 1997;9 Suppl $1: 65-9$. 
49. Bell CC. DSM-IV: Diagnostic and Statistical Manual of Mental Disorders. JAMA. 1994;272(10):828-9.

50. Gillespie LD, Robertson MC, Gillespie WJ, Sherrington C, Gates S, Clemson LM, et al. Interventions for preventing falls in older people living in the community. Cochrane Database of Systematic Reviews. 2012(9).

51. El-Khoury F, Cassou B, Charles MA, Dargent-Molina P. The effect of fall prevention exercise programmes on fall induced injuries in community dwelling older adults: systematic review and meta-analysis of randomised controlled trials. Bmj. 2013;347:f6234.

52. Vassallo M, Mallela SK, Williams A, Kwan J, Allen S, Sharma JC. Fall risk factors in elderly patients with cognitive impairment on rehabilitation wards. Geriatrics \& Gerontology International. 2009;9(1):41-6.

53. Eckstrom E, Parker EM, Lambert GH, Winkler G, Dowler D, Casey CM. Implementing STEADI in Academic Primary Care to Address Older Adult Fall Risk. Innovation in Aging. 2017;1(2).

54. Baker J, de Laat D, Kruger E, McRae S, Trung S, Zottola C, et al. Reliable and valid measures for the clinical assessment of balance and gait in older adults with dementia: a systematic review. European Journal of Physiotherapy. 2020:1-12.

55. Wittwer JE, Webster KE, Andrews PT, Menz HB. Test-retest reliability of spatial and temporal gait parameters of people with Alzheimer's disease. Gait \& Posture. 2008;28(3):392-6.

56. Delbaere K, Close JC, Taylor M, Wesson J, Lord SR. Validation of the Iconographical Falls Efficacy Scale in cognitively impaired older people. J Gerontol A Biol Sci Med Sci. 2013;68(9):1098-102.

57. Hauer KA, Kempen GI, Schwenk M, Yardley L, Beyer N, Todd C, et al. Validity and sensitivity to change of the falls efficacy scales international to assess fear of falling in older adults with and without cognitive impairment. Gerontology. 2011;57(5):462-72.

\section{Figures}
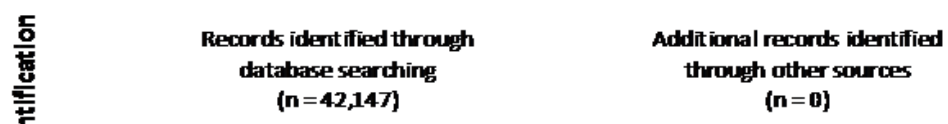

Records after duplicates remowed

(n $=20,727$ )

혿

Records screened

(n $=20,727)$

$\frac{\text { 호․ }}{\overline{\overline{5}}}$

Full-text articles assersed

for edigbility

( $\mathrm{n}=479$ )

Studies included in

quantitat ive synthesis

(meta-analysis)

(n=11)
Records exchuded

(n $=28,248$ )

Figure 1. PRISMA Flowchart

Figure 1

See image above for figure legend 
A.

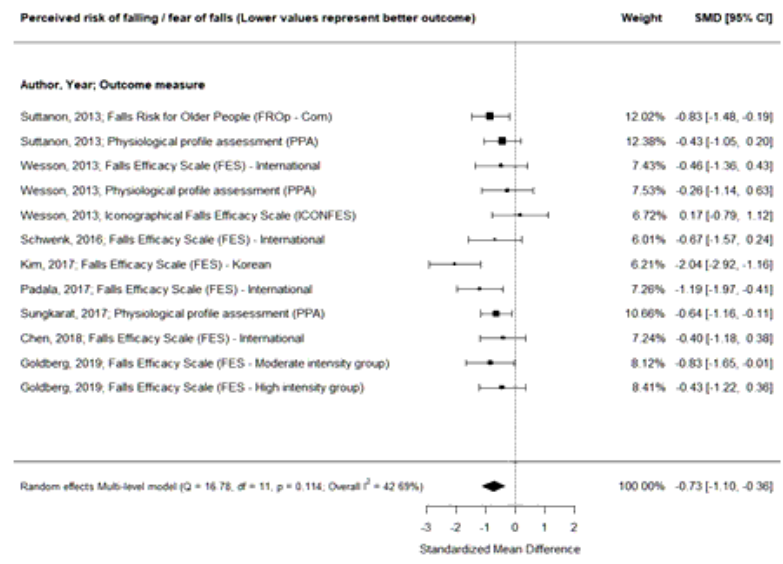

C.

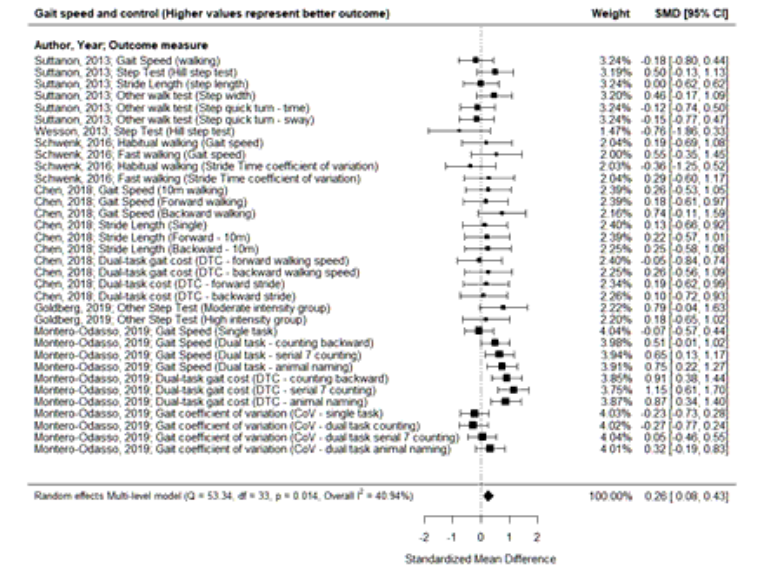

B.

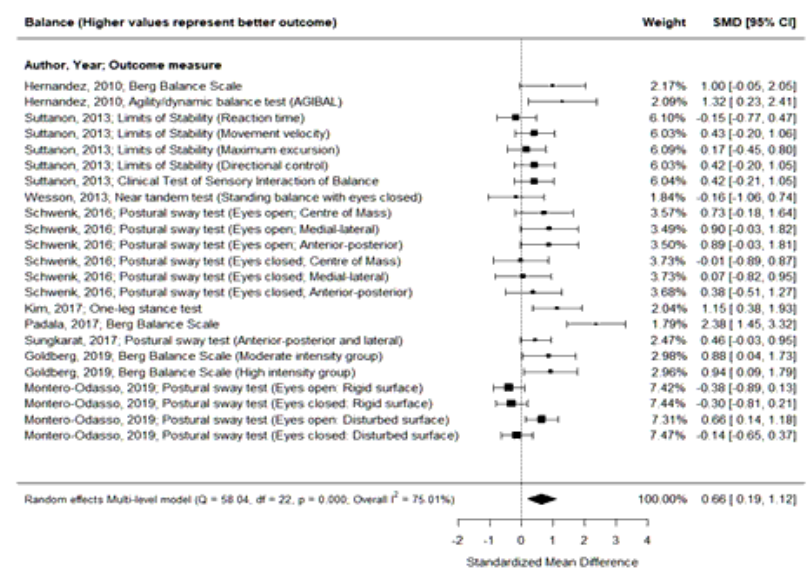

D.

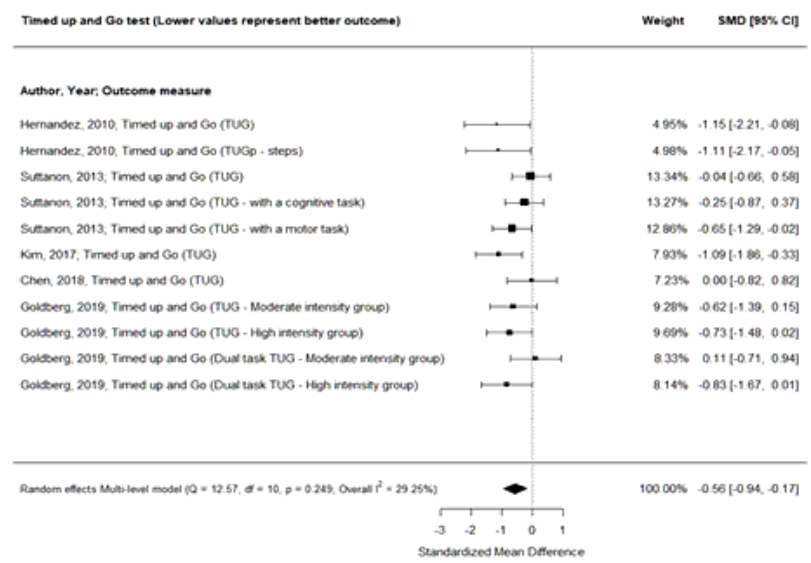

Figure 2. Effects of fall prevention intervertions on perceived risk of falk (A), balance (B), gait speed and control(C), and timed $\varphi$ p and go (D) outcomes. Weights are from random effects multi-ievel model analysis. Note: SMD = standardized mean difference, $\mathrm{Cl}=$ confidence interval

Figure 2

See image above for figure legend

\section{Supplementary Files}

This is a list of supplementary files associated with this preprint. Click to download.

- AdditionalFile1SearchStrategy.pdf

- AdditionalFile2Outcomedefinitionsandmeasurements.docx

- AdditionalFile3Characteristicsof IncludedStudies.docx

- AdditionalFile4GRADETargetedFallspreventtion.docx

- AdditionalFile5ForestPlotsforalloutcomes.docx

- AdditionalFile6SubgroupAnalysis.docx 